\title{
MOUVEMENT SENSIBLE ET CORPS ORIGINAIRE
}

\author{
Apport de Maine de Biran et Kandinsky \\ pour une phénoménologie radicale, esthétique et culturelle \\ selon Michel Henry
}

Rolf Kühn

Institut für Existenzanalyse und Lebensphänomenologie Berlin

Par la description du corps comme pouvoir originel identique à la vie transcendantale de l'ego, on peut établir, par une phénoménologie radicale ou matérielle, que la vérité phénoménalisante du corps absolument subjectif se situe dans l'immanence de l'auto-affection passible. Suivant ici surtout les analyses de Maine de Biran et Kandinsky aux yeux de Michel Henry, il faut alors montrer que la potentialité inhérente à la corporéité révèle une sensibilité charnelle qui communique avec l'univers entier et fait de lui un cosmos vivant. Cette vie cosmique, dont l'art en général - et l'art abstrait en particulier - nous fait comprendre cette réalité subjective, est l'équivalent de nos forces ou pulsions intérieures, comme on peut l'étudier, en particulier, par le mouvement de la main. Ainsi, il se trouve vérifié, par un exemple précis, que le corps propre donne effectivement accès au monde sans être réduit en lui-même à un simple instrument ou moyen transcendant, étranger à notre vie intime.

\section{Statue de rose et mobilité de la main}

Sur les représentations hindoues, on peut souvent voir des divinités ayant plusieurs bras qui signifient leur aide par l'essence de la puissance en elles qui se multiplie par tous les actes d'intervention possible. De même, les statues de Bouddha et du Christ montrent une main levée en signe de béné- 
diction ou de témoignage de leur révélation vraie, ce qui met le croyant, ou le simple spectateur esthétique, en face d'une unité de puissance qui dépasse un simple rassemblement de forces dans l'espace de l'extériorité comme horizon du regard habituel. Cela invite donc à comprendre, de manière générale, les reproductions infinies du pouvoir de la main ou son geste unique d'un salut possible comme la phénoménalisation d'un acte qui ne se réalise jamais en tant que mouvement isolé situé dans l'espace et la durée, mais comme la Potentialité ontologique originaire justement que l'on peut saisir dans le "Je Peux" fondamental dont parle déjà Husserl dans les "Idées II" et qu'il faut analyser ici en son mouvement immanent en tant que l'auto-donation de la vie au sens d'un (se) donner sans restriction.

Ce détournement du regard mondain par rapport à la main et sa capacité absolument subjective de "préhension" fut réalisé également, d'une façon réductive propre, par Pierre Maine de Biran (1766-1824) critiquant les théories de Condillac. D'après ce dernier, l'homme se trouve affecté par des sensations multiples, sans qu'il y ait en elles de moyen caractéristique pour les localiser dans un lieu défini. La thèse essentielle de ce sensualisme consiste donc à dire que la totalité de la matière de notre connaissance provient du flux d'informations correspondant à la sensation. Et comme l'a illustré Condillac par son exemple célèbre d'une statue qui acquiert peu à peu des connaissances et des facultés à partir d'une sensation initiale, le système opératoire qui effectue le traitement des sensations provient également de celles-ci. C'est en fonction de cette hypothèse que, dans son Traité des sensations de 1754, l'analyse des sensations suit, pas à pas, leurs compositions et décompositions selon la voie qui se veut naturelle, c'est-à-dire en refusant la synthèse qui, aux yeux de Condillac, part toujours d'abstractions. Le modèle de la statue qui est toute entière odeur de rose, quand elle sent la rose au début, suppose que toute théorie de l'entendement se construise à partir de l'observation externe et non pas en suivant la réflexion sur soi du sujet connaissant. Le critère du vrai, pour Condillac, est donc le fait au sens de quelque chose de logiquement indéfinissable, ce qui explique, chez ce philosophe, le finitisme métaphysique radical qui repose, en effet, sur sa conception d'une perception entièrement composée de sensations simples en nombre fini.

Le refus de la réflexion sur l'ipséité, à titre de présupposé allant de soi, trouve alors sa base, chez Condillac, dans une conception selon laquelle la conscience de soi devrait correspondre à quelque fait ou donnée empiriques. Pour y parvenir il propose, par conséquent, de considérer toute activité réflexive comme une sensation transformée. Ainsi, l'attention devient la soumission à une sensation, tandis que le souvenir marque le prolongement d'une influence sensible, puisque les idées ne forment, en ce cas, que des souvenirs de perceptions retenues par notre mémoire. Comparer et juger signifient prêter attention simultanément à deux sensations et la faculté 
d'abstraction devient, de la sorte, l'isolement d'une sensation saisie à partir d'un rapport. Mais Condillac ne se contente pas d'une simple énumération ou classification d'éléments simples comme chez les "Idéologues" ultérieurs en France, il s'efforce en plus de pénétrer les opérations psychiques jusque dans leur source, faisant de l'homme un être sensible avant tout et refusant, par là, le rationalisme cartésien des idées innées régnant encore à son époque. Et si l'on suit attentivement l'ordre de la naissance des sensations à propos de sa statue humaine fictive et sans représentation, il faut admettre que Condillac commence bien avec l'odorat, qui est le sens réceptif le plus faible, pour aboutir au sens tactile supérieur, puisque celui-ci éveille en nous activement l'idée d'un monde extérieur ainsi que la perception de notre propre corps.

En fait, la matérialité éprouvée du monde demande un contact qui apprend en même temps aux autres sens de juger adéquatement cette extériorité. Nous trouvons ici la réponse cherchée concernant la localisation des sensations multiples. Si le toucher apprend vraiment aux autres sens que leurs perceptions ne constituent pas seulement des "modifications de l'âme", comme disait en outre Maine de Biran, mais des qualités d'un monde indépendant, c'est que Condillac cherche, en effet, derrière les sensations le réel qu'elles indiquent et en lequel elles prennent place. À ce niveau, nous retrouvons finalement la thématique de la main, car elle est l'organe de sensation de la solidité. En tant que telle, la main ne révèle pas seulement le monde dans sa signifiance fondamentale pour l'homme, c'est-à-dire celle d'un être ferme et stable qui ne recule pas, puisqu'il résiste en tant que "solide", mais cette même main nous révèle aussi graduellement - par son application aux différentes parties du corps - l'être et les formes réelles de chaque partie du corps. Pour Condillac, ainsi que pour toute une tradition naïve et philosophique, la main est donc considérée comme l'instrument de la connaissance du monde et de notre propre corps. Si nous nous limitons ici, essentiellement, à l'appréhension de notre corps visible, il faut dire que toute la problématique de Condillac se meut phénoménologiquement dans un vaste cercle, car il présuppose constamment ce qu'il veut expliquer: la main qui s'applique aux différentes parties du corps, n'est-elle pas déjà le corps propre ou subjectif (Leib en allemand) qu'elle prétend découvrir? Autrement dit, avant la découverte d'un corps extérieur et objectif, il existe déjà un corps originaire qui réside dans ce mouvement même de la main en train de se déplacer avec un savoir sûr, sans égal, et que nous connaissons immédiatement, en nous, comme une praxis subjective absolue. D'où la main tient-elle ce savoir ou, pour reprendre les questions décisives de Maine de Biran, ce phénoménologue du corps subjectif avant l'heure, à l'égard de Condillac: "Comment cet instrument est-il connu d'abord lui-même?" Et 
"comment un organe mobile quelconque a-t-il été constamment dirigé sans être connu?"1

Phénoménologiquement, l'activité extraordinaire de la main pose ainsi le problème du mouvement propre qui est toujours déjà en notre possession et qui n'est pas connu par le regard de la réflexion ou de la visée de l'intentionnalité se dirigeant sur lui. S'il n'y a aucune distance entre le.mouvement de la main et nous-mêmes, la phénoménalité de cette main ne relève plus alors de la transcendance comme chez Condillac ou chez d'autres philosophes du corps jusqu'à Merleau-Ponty. Bien au contraire, cette main nous fait découvrir la subjectivité absolue de notre corps propre qui - avant d'être l'ensemble de nos pouvoirs sur le monde - constitue la source de tout pouvoir en sa potentialité originaire, comme nous l'avons déjà constaté en partie. Avec Maine de Biran, une telle analyse de la main conduit donc spécifiquement au problème central de la connaissance originaire de notre corps, dont l'intégralité et la particularité dépassent toute connaissance transcendante des choses extérieures.

Si le mouvement de la main est connu dans le sens où il peut être dirigé par nous instinctivement ou selon notre désir, cette connaissance ne relève précisément pas d'une constitution au sens husserlien, mais plutôt d'un $s a-$ voir primordial pratique qui n'est appréhendé en aucun monde phénoménal objectivé ou transcendant. L'immédiateté de ce savoir primordial fait alors appel à une connaissance qu'il faut bien nommer, avec Maine de Biran, une expérience interne transcendantale se confondant avec l'être même de ce mouvement en tant que 1' "effort", comme il dit. Il n'y a aucune distance, pour l'exprimer autrement, entre' la "connạissance" et l'être même de ce mouvement de la main, de sorte que l'absence de tout champ ou milieu transcendants exclut également, par essence, toute notion d'un déplacement à l'intérieur d'une spatialité objective, pour ne laisser subsister que l'immanence d'un mouvement originaire ou purement subjectif en sa réalité interne ou transcendantale même. S'il faut ajouter que ce mouvement est "en mon pouvoir", il faut alors réduire, en même temps, toute référence mondaine à mes prises de pouvoir ou à mes manipulations des choses, ce qui revient à affirmer que le pouvoir de la main est son propre pouvoir en tant que tel - ce pouvoir, autrement dit, par lequel la main se meut d'elle-même.

Lorsque Maine de Biran ajoute que "l'âme ne pense pas d'avance à l'objet de son vouloir ou aux instruments qui doivent l'exécuter et qu'elle ne

1 Mémoire sur la décomposition de la pensée (Oeuvres, t. III, éd. P. Tisserand), Paris, 1932, p. 4 et 7. Cf. aussi: De l'existence. Textes inédits (éd. H. Gouhier), Paris, 1966, p. 25 sqq., ainsi que M. Henry, Philosophie et phénoménologie du corps. Essai sur l'ontologie biranienne, Paris, 1965, p. 107 sqq.; Incarnation. Une philosophie de la chair, Paris, 2000, p. 195 sqq.; Phénoménologie de la vie t. I, Paris, 2003, chap. VIII: Le problème du toucher. 
connait pas", 'il convient d'entendre cette proposition en un double sens qui, en réalité, ne fait qu'un. Le mouvement n'est nullement un "instrument" entre le moi et le monde, ce qui signifie en toute radicalité phénoménologique, au sens de Michel Henry, que c'est l'ego lui-même qui agit directement sur le monde et sans l'intermédiaire d'un "corps", puisque cet ego lui-même ou, identiquement, le mouvement qui se déploie à partir de son pouvoir subjectif absolu et intérieur est ce corps. Ou encore, pour formuler ici nettement la problématique centrale d'une phénoménologie matérielle radicale de la Vie, on peut affirmer également que, en ce savoir primordial de la mobilité comme auto-mouvance, la main est l'ego même, et cela dans la mesure où celui-ci est vie, autrement dit la vie d'une subjectivité transcendantale unique dans toutes ses modalités et dans toutes ses déterminations. ${ }^{2}$

Reprenons encore une fois, à cet endroit, les représentations habituelles du corps objectif (Körper en allemand) comme instrument ou intermédiaire, pour les confronter à l'enseignement fulgurant de Maine de Biran. On dit couramment que le corps constitue l'instrument de mon action sur le monde et que, grâce à son intervention, je peux changer ce même monde dans un sens voulu. Cela va certainement de soi pour la perception quotidienne, mais si j'exécute des mouvements sans y penser, ce n'est nullement que ces mouvements soient mécaniques ou même inconscients. De telles théories font perdre l'essentiel du mouvement propre qui, en son être ontologique même, appartient phénoménologiquement tout entier au domaine de la transparence immanente absolue de la subjectivité sensible ou affective. Comme nous l'avons déjà vu au sujet du savoir primordial de la main vivante comme praxis subjective, il n'y a aucun intermédiaire entre 1'“âme" ou la vie et le mouvement en tant que tel, étant donné que toute distance entre eux se trouve abolie par l'immanence de l'auto-affection du pouvoir subjectif qui, justement, "sait" immédiatement ce qu'il faut faire. L'idée d'un intermédiaire est, par conséquent, une fiction de la pensée réflexive en tant que telle. Celle-ci se donne des représentations spatiales et causales, puisqu'elle s'accomplit elle-même au milieu de la transcendance d'un "champ de conscience" (Husserl) qui, par définition, est la phénoménalité extérieure de l'extension et de la juxtaposition - ou de la temporalité (Heidegger) en dernière analyse.

$\mathrm{Si}$, précisément, nous n'avons pas besoin de penser à notre main ou à notre corps intégral pour accomplir des actions, c'est que celui-ci ne s'offre

1 Essai sur les fondements de la psychologie et sur les rapports avec l'étude de la nature (Oeuvres, t. VIII, éd. P. Tisserand), Paris, 1934, p. 194. Il faut signaler, pour accéder maintenant plus facilement aux écrits de Maine de Biran, qu'il existe, depuis 1987, une édition critique de ces "Oeuvres" sous la direction de F. Azouvi chez Vrin à Paris.

2 Pour une vue différente de l'ego biranien, en comparaison avec Descartes, cf. M. Merleau-Ponty, L'union de l'âme et du corps chez Malebranche, Biran et Bergson (éd. J. Deprun), Paris, 1968, pp. $62-78$. 
pas dans la lumière ek-statique du monde où il ne serait qu'un "moyen". Il s'offre lui-même plutôt par un accomplissement spontané qui est l'oeuvre phénoménalisante de la vie originaire elle-même. Dans cette vie, nous le savons par les analyses magistrales de Michel Henry, l'auto-mouvance, la corporéité et 1'ego ne désignent, finalement, que la même chose, sans se dissoudre pour autant dans la nuit d'un "inconscient" quelconque, car cette vie phénoménologique pure, avec ses manifestations modales, est l'être réel même de notre ego transcendantalement subjectif et absolu. Par contre, l'instrument et le moyen font toujours appel à un rapport distant d'utilisation, c'est-à-dire à quelque chose d'autre qu'eux-mêmes. Une telle éventualité n'est point exclue, mais si le sujet ou la pensée "se servent" du corps, ce sujet en possède alors une connaissance différente, autrement dit une connaissance transcendante. Ou, pour le dire avec Maine de Biran lui-même: "Nous connaissons très bien les forces motrices de notre corps qui est le moi, et toutes les forces impulsives extérieures sont nettement conçues d'après ce modèle [...]. L'âme s'aperçoit immédiatement dans son action et sans représenter rien hors d'elle-même ou du moins hors du terme organique sur qui elle agit ou exerce sa force motrice."1

C'est à ce niveau transcendant pourtant que se situent la plupart des philosophies traditionnelles du corps qui en proposent une représentation mondaine, sans prêter attention à l'Apriori décisif qui est la nature vivante du corps, comme il faut le souligner pour répondre à une radicalité phénoménologique exigée par cette manifestation unique de la corporéité sans pareil. ${ }^{2}$ Car penser la main au niveau de sa finalité intentionnelle et de son instrumentalité transcendante n'est point agir, puisque l'action ne relève d'abord jamais d'une connaissance thématique préalable, mais seulement de la force ou de la praxis intérieures en tant que ce déploiement d'une potentialité qui, quant à elle, renferme tout savoir nécessaire à la vie. Bien sûr, il ne s'agit nullement de refuser les connaissances transcendantes qui peuvent être thématisées à partir de l'anatomie, de l'haptologie, etc., c'est-à-dire par les sciences naturelles en général. Toutefois, il faut rester conscient du fait que l'énumération ou la description de ces acquisitions manuelles constituent un

1 De l'existence, p. 41.

${ }^{2}$ Pour une discussion phénoménologique élargie à ce sujet, cf. aussi J.-F. Courtine, Heidegger et la phénoménologie, Paris, 1990, pp. 283-303: Donner/prendre: la main; J. Derrida, Le toucher - Jean-Luc Nancy, Paris, 2001. Nous avons exposé ces différents enjeux phénoménologiques dans notre ouvrage: Pierre Maine de Biran - Ichgefühl und Selbstapperzeption. Ein Vordenker konkreter Transzendentalität, Hildesheim-Zurich-New York 2006 (avec bibliographie complète sur le biranisme). Cf. également S. Laoureux, L'immanence à la limite. Recherches sur la phénoménologie de Michel Henry, Paris, 2005, chap. II: Les figures du "contenu resistant" biranien dans la phénoménologie matérielle. On y trouve le jugement injustifié d'un transcendantalisme à l'outrance de M. Henry doublant, pour ainsi dire, l'empirique. 
phénomène d'un autre ordre que ce mouvement réel ici en question et dont il ne peut exister de vision théorique.

\section{Tracé de main et phénoménologie esthétique}

C'est la création esthétique qui nous rapproche certainement le plus de cette seule réalité subjective absolue contenue, par exemple, dans une ligne tracée par une main sur une surface. Pour le peintre Kandinsky, ce phénoménologue pratique et théorique extraordinaire des formes et des couleurs, tout phénomène peut être considéré de deux façons: en sa manifestation extérieure et en son intériorité radicale. ${ }^{1}$ Ainsi, la force concentrique enfermée dans un point qui subit une autre force agissant sur lui de l'extérieur, est poussée dans une autre direction, et cela aussi longtemps que cette force ne change pas. Une ligne est l'effet immédiat de cette force qui intervient, et il faut même dire, plus adéquatement, que cette force devient véritablement la ligne et se déploie ainsi en une direction donnée. Par une telle ligne, une tension liée à son mouvement est alors introduite dans un dessin qui, par la composition d'autres éléments graphiques, telles les couleurs, peut devenir de cette manière une véritable symphonie des pulsions subjectives. Car la tension "visible" en une ligne "extérieure" du dessin n'est rien d'autre que le transfert de la force subjective avec laquelle le peintre a répondu à une affectivité "intérieure". Ou, plus précisément encore, cette ligne qui ne représente rien figuralement et qui n'emprunte rien au monde est, en son essence même, cette affectivité éprouvée qui suscitera, chez le spectateur esthétique, une résonance affective du même ordre.

Le génie propre de Kandinsky consiste alors en une étude minutieuse de ces résonances qui ne sont pas laissées au hasard. Ainsi, pénétrant plus avant dans cette phénoménologie de la ligne tracée par une main charnelle, on dira qu'une force unique agissant de manière constante produit une droite. L'action simultanée ou successive de deux forces engendrera la ligne courbe ou brisée. Il est évident que l'inflexion de la courbe dépendra de la puissance chaque fois déterminée du mouvement actif qui produit une droite. Le temps et l'intensité interviennent naturellement de la même manière pour décider de la longueur et de l'angle de chaque segment composant une ligne brisée. Tout cela se trouve accompli par la main dont l'être le plus originaire relève de la vie transcendantale de l'ego, comme nous l'éprouvons à partir de la passibilité qui est notre chair même au niveau d'une sensibilité esthétique et pratique. Si nous admettons maintenant, en plus, que les lignes d'un

1 Cf. Punkt und Linie zu Fläche. Beiträge zur Analyse der malerischen Elemente, Berne, 1973, p. 13 (traduction française: Point-ligne-Plan, Paris, 1970). Cf. également M. Henry, Voir l'invisible. Sur Kandinsky, Paris, 1988, p. 88 sqq.; Phénoménologie de la vie, t. IV: Sur l'esthétique et la religion, Paris, 2004. 
graphisme des forces ou pulsions invisibles de cette vie subjective, deviennent manifestes par le mouvement vivant de la main, nous pouvons comprendre plus facilement que le projet révolutionnaire de Kandinsky en peinture consistait à exprimer la vie "intérieure" à l'aide de lignes composées avec ou sans couleurs. Le principe "abstrait" de cet art est donc acquis définitivement pour l'orientation ultérieure de la peinture moderne: chaque pulsion forme la trame de la subjectivité et reçoit son équivalent immédiat dans une forme linéaire donnée qui ne se réfère plus à une représentation des choses dans l'espace.

Maine de Biran rejette l'idée d'un corps-instrument et, avec Kandinsky, l'idée de l'image-représentation se trouve abolie de la même façon en faveur du seul pathos immanent du sujet vivant ou charnel. La représentation d'une ligne tracée suppose, en effet, la mise en rapport de déterminations ontologiques susceptibles de consonance, c'est-à-dire une sorte de "parallélisme" homogène entre le visible (l'image représentée) et l'invisible pratique (pulsion subjective). Mais si tout trait effectué par la main vivante "exprime" d'abord la passibilité de la vie donnée en tant qu'étreinte pathétique d'une force en elle-même et par elle-même pour s'emparer de son propre pouvoir, il n'y a pas seulement une homogénéité entre le tracé et cette force invisible - il existe plutôt une identité entre les formes linéaires et les pulsions intérieures de notre vie phénoménologique pure. D'après Kandinsky toujours, interprété par Michel Henry, il n'y a, pour l'art abstrait, aucun mystère d'analogie, d'affinité ou de correspondance qu'il faudra élucider de manière causale, puisqu'en fonction de la réciprocité phénoménologique entre l'intérieur et l'extérieur, c'est la même Force que nous éprouvons sous la forme charnelle de son pathos et en tant que pro-duction effective de la ligne. Par conséquent, l'idée dépassée d'une représentation différente de la réalité imaginée esthétiquement nous conduit également à l'abandon de toute théorie d'une mimesis dans la tradition grecque qui voudrait faire pré-exister cette vérité esthétique dans la sphère d'un monde archétypique.

La main, rendue à sa seule puissance charnelle intérieure, est alors capable, sans aucun intermédiaire théorique ou général, de "transférer" le lyrisme et le drame que nous éprouvons en nos vies dans le jeu "abstrait" des lignes droites et brisées. Et nous pressentons d'autant plus la profonde vérité de certains dessins ou tableaux de Kandinsky que, lorsqu'ils font voir une sorte d'affrontement cosmique des forces, nous y retrouvons projetées nos propres énergies de vie. Une telle vision est rendue possible grâce à la libération, due à l'art abstrait, qui n'imite aucun tracé visible en tant que contour d'un objet réel, comme on peut le voir, en outre, sur les tableaux de Klee ou 
de Rothko. ${ }^{1}$ Ce n'est pas seulement la main tremblante qui, par exemple, exprime l'émotion de la peur, c'est la main tout entière, la main comme telle, c'est-à-dire en tant que notre chair passible, qui transmet à l'univers mort sa vie pour en faire une réalité vivante et spirituelle au sens d'un cosmos unique. Cette réalité est d'abord celle de notre impressionnabilité qui s'auto-affecte transcendantalement en notre immanence, de telle sorte que la sensibilité n'est pas seulement - comme chez Condillac et d'autres - un système d'informations sensitives, mais vraiment "la chair de l'univers". Car le toucher vivant fait vibrer les choses au rythme de nos propres vibrations intérieures.

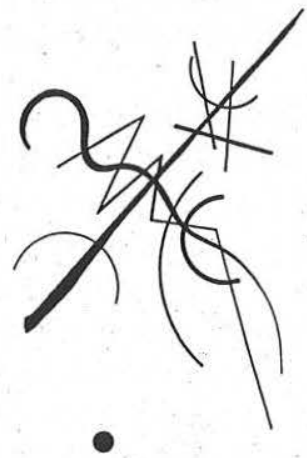

Wassily Kandinsky

Tension diagonale avec contre-point

(Point-Ligne-Plan, figure 20)

L'esthétique de l'art abstrait et, en dernière analyse, de toute peinture nous reconduit ainsi à l'essence de l'émotivité et de l'affection qui, dans leurs sonorités intérieures, sont voilées habituellement par des finalités utilitaires jusqu'à cette libération des énergies vives. Mais une foịs que le regard en quête de l'utile seul a été abandonné, l'appel esthétique s'adresse à la totalité des forces de la vie pour qu'elles puissent s'exercer dans leur plénitude pathétique potentielle. Autrement dit, c'est la subjectivité de l'ego absolu qui est rendue à la véritable vie charnelle: celle qui vibre, justement, dans sa chair affective pour faire vibrer l'univers en l'habillant des qualités subjectives sensibles que l'on dit à tort "secondaires", surtout après Galilée et sa réduction de l'univers à des formes géométriques. Car, pour suivre

${ }^{1}$ Pour la peinture classique également analysée en fonction d'une phénoménologie radicale, cf. aussi G. Dufour-Kowalska, Caspar David Friedrich. Aux sources de l'imaginaire romantique, Lausanne, 1992. 
toujours notre exemple, la ligne tracée par la main est reliée à tout un univers où nous sommes chez nous à cet instant, c'est-à-dire libres d'exécuter nos mouvements les plus intérieurs qui sont les forces ou les pulsions ainsi que les efforts et désirs mêmes de notre vie. De cette façon, on conçoit aisément l'impact d'une telle expérience esthétique, car plus le mouvement de la main s'affine, plus il devient apte à participer à l'accroissement de la Potentialité vivante en nous en tant qu'archi-révélation de toute manifestation phénoménologique comme telle. La main elle-même devient ainsi tout artistique pour exprimer, par ses tracés et ses touches qui rappellent également la musique, des nuances de plus en plus fines de la vie de nos sentiments, ce qui provoque une joie de plus en plus grande, une jouissance qui est celle de l'étreinte passible de la vie elle-même - et par cette voie l'art réalise la maîtrise esthétique suprême, et même éthique, du corps subjectif par lui-même et en lui-même.

Cette intensification ou libération de la potentialité vivante intérieure que nous avons également analysée comme l'essence phénoménologique de toute culture ${ }^{1}$ constitue, en tant que jouissance créatrice, le sentiment que la vie possède d'elle-même et qui justifie, à partir de cette immanence chaque fois concrète, la détermination modale d'une ligne en son tracé particulier. Car l'expérience ou l'appréhension internes des forces qui engendrent ce tracé est exactement le sentiment de la vie éprouvée qu'on veut exprimer. Voilà aussi la raison pour laquelle il n'y a aucune règle préétablie pour l'art de dessiner, car si sa source réside dans le seul pathos d'une force vivante, la "nécessité intérieure" (innere Notwendigkeit), selon Kandinsky, abolit toute norme objective ou idéale. À ce titre, le mouvement de la main créant une ligne nous met en présence d'une liberté infinie et d'une non-liberté primordiale qui échappent, par exemple, à la vue dialectique de Hegel sur l'ob-jectivation qui réaliserait seule l'en-soi intérieur. ${ }^{2}$ Car, au-delà de la diversité infinie de tonalités intérieures et, partant de la liberté sans borne de les traduire dans une composition esthétique, il existe aussi une nécessité indépassable de la passibilité immanente où la vie est rivée à elle-même pour être elle-même. Cette auto-affection de la vie en son étreinte phénoménologique éternelle qui constitue son archi-révélation même, est le fondement de toute potentialité, comme nous le savons maintenant; et, de cette façon, n'importe quelle force vivante repose sur cette archi-révélation de la vie charnelle, c'est-à-dire sur la venue à la vie de 1'“être" lui-même. Sans cette étreinte de soi par soi, sans cette essence et ce contenu mêmes de la vie en sa parousie intérieure, il n'y aurait aucune potentialité qui soit le pouvoir réel d'une force se saisissant comme en-soi et pour-soi simultanément. Et, par consé-

1 Cf. notre ouvrage: Ästhetische Existenz heute. Zum Verhältnis von Kunst und Leben, Fribourg/Munich, 2006.

2 Cf. Phänomenologie des Geistes (Gesammelte Werke 9), Hambourg, 1988, pp. 69 sqq. 
quent, il n'y aurait aucune corporéité au sens analysé ici, puisque celle-ci consiste en la vie transcendantale même de l'ego absolu au niveau de la subjectivité affective qui "souffre" sa passibilité en "se réjouissant" de la vie qui lui est donnée dans leur ipséité réciproque. ${ }^{1}$

La création d'une typologie graphique par Kandinsky est donc autre chose que la création d'un répertoire simplement à imiter. Appuyé sur la diversité charnelle ou sensuelle de sa vie, sur les modalisations indéfinies de ses vécus affectifs, l'artiste dessine autant de lignes qu'il est capable d'en éprouver. Or, le résultat qui en découle n'est pas seulement un immense domaine "formel" où chaque combinaison de forces est donnée avec son affect propre. Le jeu de ces forces instinctives" est, en même temps, l'historial méta-généalogique de notre vie, c'est-à-dire la possibilité transcendantale pour tout individu empirique. Si nous laissons de côté ici le problème de la composition proprement dite d'une peinture achevée, il faut néanmoins souligner, une fois de plus, que l'oeuvre de la main vivante réalise l'essence même de la culture. Car chaque assemblage inédit de lignes expérimente de nouvelles tonalités fondamentales de la vie. Par cette voie, des émotions que les hommes n'avaient peut-être encore jamais éprouvées, élargissent à l'infini la sphère des expériences concevables et permettent de considérer la culture comme l'archi- et l'omni-réalisation de la vie. Donner à sentir tout ce qui peut être senti, faire éprouver tout ce qui peut être éprouvé, telle est l'ambition phénoménologique de la vocation artistique de la main qui nous révèle, ensemble avec les autres sens, toutes les forces de notre être, autrement dit, le pouvoir ontologique même de notre corporéité pathétique ou charnelle.

\section{Cosmos et "chair du monde" comme chair vivante}

Précisons, enfin, le lien unissant cette potentialité, cette auto-révélation de la vie immanente de l'ego, à la sensibilité-chair qui fait du cosmos, à partir de la vie invisible, cet ensemble déjà évoqué de forces vivantes. Par principe, l'art nous apprend que les qualités sensibles ne doivent pas être réduites à de simples signes ou caractères d'une réalité étrangère à notre vie, mais que toutes ces impressions et sensations forment, avec les émotions et les sentiments, de véritables modes de la vie subjective naturante. En arrachant ainsi les formes linéaires et les couleurs aux significations du monde objectif, la peinture abstraite ou non référentielle rend à la Nature cosmique son essence intérieure. Cette nature originelle et subjective, dynamique et char-

Pour une recherche plus développée concenrnant la pensée intégrale de M. Henry, cf. également notre ouvrage: Leiblichkeit als Lebendigkeit. Michel Henrys Lebensphänomenologie absoluter Subjektivität als Affektivität, Fribourg/Munich, 1992. 
gée d'émotions, dont l'essence éprouvée est la vie charnelle même, constitue le cosmos, la terre et le ciel avec ses planètes et ses étoiles. Tous les êtres, qui appartiennent donc à ce cosmos, font partie de lui en tant qu'éléments sensibles ou "corpspropriés", pour emprunter un terme propre à Michel Henry, ${ }^{1}$ c'est-à-dire qu'ils ne se manifestent phénoménologiquement que dans une sensibilité originaire et par elle. Et suivant la mesure de cette charnalité impressionnelle ou affective, ces choses sont autant de subjectivités au sens strict qui remplissent le monde de résonances et exercent sur l'âme une action spirituelle dont elle retentit. Il n'y a donc pas de matière qui ne soit une tonalité spécifique ou "cosmos spirituel" (geistiger Kosmos) au sens de Kandinsky.

Bien entendu, l'art - comme toute autre activité en tant que praxis subjective - reste un processus de culture avec ses lois particulières, où l'on peut détecter une "typologie" et "imitation" possibles avant le seul déroulement historique des événements. ${ }^{2}$ De plus, il existe, dans l'art et dans la nature, les mêmes principes fondamentaux qui sont la juxtaposition et l'opposition et dont dérivent, pour la composition esthétique, les parallèles et les contrastes. Par là, une même loi générale de l'univers, mais sensuellement concrète, se dessine. D'après Kandinsky, elle est la synthèse de l'intérieur et de l'extérieur en leur fondement ultime. Cette scission phénoménologique d'une archi-facticité rigoureuse, sur laquelle repose l'immanence et la transcendance, avec leur ordre analytique respectif, est néanmoins appelée une unité de régulation dernière, renvoyant ainsi à la subjectivité des éléments dont la sonorité décidera de leur répartition et de leur assemblage. Car si chaque élément cosmique possède sa sonorité propre, cela veut alors dire qu'il subit l'épreuve de lui-même dans une impression qui est celle de sa vie. De cette manière, il n'y a pas de monde sans sensibilité charnelle, sans cet ensemble de sensations affectives qui forment le tout de notre sensibilité - cette "chair" même de l'univers qui est entièrement vivant. Cette nature en sa subjectivité, qui est celle de la couleur de l'objet et sa forme, n'advient que dans le flux matériel de notre vie, à savoir comme sentiment de la nature et, de façon générale, comme intériorité radicale de toute extériorité possible. Si l'artiste accomplit cette oeuvre par sa main, c'est-à-dire toujours par son corps absolument subjectif, il réalise, par là, la révélation de la vie selon sa volonté propre. La dignité totale est ainsi rendue à ce corps retrouvé dans la vie, puisque c'est lui qui procède à cette archi-révélation de la vie. Et en conduisant celle-ci à son telos par sa chair sensible et sensuelle, le corps veut faire sentir le maximum des forces à l'œuvre, à savoir finalement un univers entier qui est le "corrélat" total et entièrement pratique promis à ce corps-

La Barbarie, Paris, 1987, p. 80 sq.

${ }^{2}$ Cf. Radicalité et passibilité. Pour une phénoménologie pratique, Paris, 2003, chap. 12 sur la culture, ainsi que notre $4^{\text {ième }}$ paragraphe suivant. 
-ego, grâce à son affection intérieure, qui se situe avant toute temporalisation ek-statique au sens de la phénoménologie historique.

Si ces "descriptions" doivent beaucoup à l'oeuvre phénoménologique et esthétique de Michel Henry, c'est parce qu'il a su ouvrir, par sa phénoménologie radicale de l'immanence auto-affective en tant que l'essence de la manifestation, des voies entièrement nouvelles pour une analyse concernant la corporéité "hylétique", c'est-à-dire s'auto-sentant et "sensuelle" en ce sens. ${ }^{1}$ Elévé au juste rang d'une vérité phénoménalisante primordiale, le corps charnel n'est plus une vérité secondaire ou seulement instrumentale, mais bien le principe même d'une ontologie vivante, à savoir l' "être" advenant comme son propre auto-apparaître par l'auto-donation de la vie. Sur le plan de la possibilité ontologique constitutive de la réalité phénoménologique, le corps - en tant que ce "Je Peux" immanent fondamental que je suis est entièrement "potentialité", comme nous l'avons souligné. Et c'est en cette Potentialité réelle que reposent, en effet, tous les pouvoirs de notre corps subjectif, autrement dit en cette essence ou matérialité phénoménologiques qui permet originairement l'accès plénier au monde. Celui-ci, en fait, ne m'est jamais donné par un "acte" isolé de voir ou de toucher, etc., mais seulement par la possibilité pratique de pouvoir le sentir aussi souvent que je le veux, et cela grâce au divers sens qui impliquent ainsi une habitualité transcendantale ayant le statut d'une mémoire purement charnelle. Et, comme terme indéfiniment évocable, ce monde se donne sous la condition concrète d'un mouvement incarné des sens qui, comme nous venons de le voir, constitue l'être phénoménalisant même de mon corps propre. La possibilité de se mouvoir n'est donc pas liée à un pouvoir particulier, mais au corps originel en tant que tel, c'est-à-dire à l'hyperpuissance d'une archi-corporéité qui déploie son essence comme identique à cette dernière. À l'instar de tout ce qui est vivant, notre corps parvient d'abord à soi dans cette archi-corporéité qui est la vie comme chair en son auto-affection ipséisante ou en son étreinte de passibilité abyssale. C'est également la raison pour laquelle la capacité permanente d'accès au monde reste identique à la vivification du cosmos, décrite ci-dessus comme réalité phénoménologique de la sensibilité affective comme "chair du monde" ou, pour le dire encore autrement, comme l'œuvre d'une liberté qui puise son pouvoir-faire en une passibilité charnelle avant toute liberté.

$\mathrm{Si}$ nous contemplons ici, encore une fois, notre main en tant que ce pouvoir radicalement subjectif de préhension en général, nous pouvons dire, en guise de conclusion, que sa connaissance pratique du solide est le principe même de la re-connaissance de tous les objets possibles à saisir et à tracer par des mouvements. Et puisque ce mouvement de saisir et de parcourir les

Voir ici surtout, pour la méthode phénoménologique en question, également de M. Henry: Phénoménologie matérielle, Paris, 1990. 
bords du solide est toujours le même en tant que pouvoir vivant, notre corps propre forme, à partir justement de cette potentialité originaire, cette mémoire archaïque du monde évoquée auparavant. Car l'accomplissement de la puissance mobile sait ce qu'il fait et se reconnait en tant qu'il se sait lui-même dans l'immanence d'une hyperpuissance originaire. Loin d'être l'Inconscient, ${ }^{1}$ l'essence de la puissance est donc la première à apparaître en tant que cette capacité de la vie de s'éprouver soi-même. Le principe de cette mémoire n'est alors ni représentatif ni ek-statique; il est l'archi-corporéité elle-même, puisque la mémoire aussi est d'abord un pouvoir appartenant au "rassemblement" qui se trouve toujours déjà accompli en nous avant toute compréhension existentiale au sens ontologique de la Sammlung heideggérienne par l'être. Un tel accomplissement du rassemblement intérieur ou méta-généalogique de notre "histoire" individuelle comme historialité affective est identique à cette archi-révélation de notre charnalité originaire dont nous avons parlé, car c'est ici que se situe l'essence même de notre être vivant par l'éternelle étreinte avec soi, autrement dit en sa passibilité affective qui demeure l'origine phénoménalisante de tout pouvoir subjectif réel. Ou, pour donner encore une fois la parole à Maine de Biran qui a inspiré, en partie, cette analyse: "Le durable d'une force qui a la puissance de vouloir et d'agir est compris ou enveloppé dans le moi phénoménal qui s'aperçoit immédiatement dans l'effort et l'action et n'a pas besoin d'être un autre pour se connaître."

\section{L'auto-mouvement de la vie et la culture}

Si nous achevons ici notre analyse de la phénoménologie matérielle de la vie pour préciser encore, sur le plan culturel, la question du passage pratique de la vie immanente au monde résistant ou transcendant, nous pouvons dire que l'objet de la culture reste toujours le même, à savoir faire sentir la Force de la même Vie dans notre besoin de vivre en toute circonstance. La peinture ou la littérature se servent de l'imagination pour créer une oeuvre culturelle, tandis que la philosophie et d'autres sciences utilisent le concept et l'analyse. Mais la phénoménologie connaît justement aussi la méthode de la "libre fiction" qui est proche du traitement d'un sujet littéraire par l'imagination, où des situations variées peuvent dévoiler des composants essentiels ou métagénéalogiques de la "nature humaine" tel que le besoin en son rôle fondamental. Mais la question de la vie reste nécessairement décisive dans les deux cas, car si la phénoménologie radicale de la vie éclaire en

${ }^{1}$ Pour l'analyse de cette notion, cf. surtout M. Henry, Généalogie de la psychanalyse. Le commencement perdu, Paris, 1985, ainsi que R. Bernet, L'inconscient entre représentation et pulsion (Freud, Husserl et Schopenhauer). Philosophie 50 (1996), pp. 66-83.

${ }^{2}$ De l'existence, p. 38 sq. 
effet un autre mode plus profond et plus ancien de la phénoménalisation que l'intentionnalité transcendante, la culture n'est pas seulement la generatio de cette même vie dans l'ordre temporel. Elle est en plus la narration du pathos ou de l'auto-mouvement de l'autoaffection en sa Parousie, soit en tant que littérature ou en tant que peinture et musique. "Narrer le pathos" place alors une telle analyse du besoin, et de son passage à la représentation comme éveil sensible, au centre de l'imagination qui implique 1'"ouverture" de la vie au monde résistant en tant que l'auto-objectivation de cette même vie, car cette narration pathétique est en même temps toujours la narration du besoin et par celui-ci. ${ }^{1}$

Or, si le propre de la Vie est son auto-saisissement strict où l'acte et le contenu restent toujours la même vie affective en sa passibilité éprouvée, et cela avant toute extériorisation et ekstase dans l'épreuve de la résistance du monde, la phénoménologie de cette vie immanente ne peut pas "faire voir" cette vie en tant qu'objet thématique, comme l'impose pourtant la phénoménologie historique depuis Husserl. Une nouvelle phénoménologie radicale ou pratique, à laquelle se trouve consacré notre analyse, peut seulement indiquer la condition absolue de tout apparaître qui est l'auto-apparaître passible dont la matière concrète est l'Affectivité transcendantale. Et cette chair affective, impressionnelle ou sensuelle, on la trouve, en tant que besoin ou substance phénoménologique purs, en tout sentiment, de sorte que la "narration culturelle" peut essayer de se saisir de cette chair au moyen de récits imaginaires qui s'apparentent alors, par ce fait même, de la Parole silencieuse de la Vie en sa certitude absolue de son s'éprouver. Si la pensée manque la vie par son principe intentionnel de distance et reste ainsi vision, théorie ou objectivité, il incombe d'autant plus nécessairement à la culture, en tant que génération d'oeuvres, de créer justement par l'imagination un autre "espace" et un autre "temps" que la spatio-temporalité objective ou géométrique. Le besoin, en tant que présence pure de la vie s'affectant absolument par elle-même, peut être par là ce "lieu" différent de tous les lieux mondains, puisque son site originaire (Sitz im Leben) reste la vie seule. Et sa temporalité propre est celle de l'Historialité immanente des tonalités affectives à partir de leur naissance dans la vie jusqu'à leur éveil sensible au monde résistant en passant par la transposition de la pro-duction d'images. La narration de ces images hors lieux et hors temps peut, à ce moment, parler de la vie même, car ces tonalités-images en tant que formes, couleurs, sons, mouvements, rêves, hallucinations etc. sont le langage, pour ainsi dire "naturel", de cette vie invisible.

Cela implique que seule la vie se "connaît" directement elle-même, tandis que toute philosophie qui veut la saisir emploie des substitutions telle 
que l'Urimpression de la conscience intentionnelle ou le Maintenant (Jetzt) de l'irruption temporelle chez Husserl qui se dirige finalement vers des essences eidétiques dont la typologie devrait permettre une science en général. Au contraire, pour une phénoménologie vraiment radicale, cette impossibilité même devient "indice" de l'auto-révélation de la vie en elle-même, en tant que Nuit, qui demande une contre-réduction, sans tomber pour autant dans un romantisme ou irrationalisme quelconques d'un inconscient ayant le caractère d'une force vitale hypostasiée ou d'un inconscient au sens de Freud. Ainsi, une littérature ou une peinture qui veulent parler de cette Nuit-Révélation telle que chez Novalis par exemple, ou sur les tableaux "mystiques" de Marc Rothko et les dernières oeuvres de Kandinsky, ne peuvent le faire qu'en faisant ressentir le pathos pur de la vie en son auto-étreinte où rien du monde ou du cosmos, c'est-à-dire du manifesté, ne soit perdu. De cette façon, la tâche de la culture, en son intégralité et compossibilité des arts comme également en tant qu'assomption axiologique du savoir pratique quotidien (à savoir travail, érotisme, habitation, fêtes et souffrance), englobe en principe toutes les activités humaines pour ne rien laisser hors de l'apparaître en tant que généré par la vie qui reste besoin d'elle-même en tous ses modes sensibles. Par cette voie, la culture devient concrètement la narration de la Vie oublieuse d'elle-même qui ne se montre jamais en son Origine, dans laquelle elle s'engouffre pourtant pour s'y emparer de soi-même seulement sans aucune altérité pensable. La pensée habituelle interprète cette Nuit ou cet Abîme de l'Oubli originel de la Vie absolue comme un contenu opaque qui cependant "illumine" cette pensée même en tant que cogitatio vivante, soit au niveau de la conscience naturelle ou dans la réflexion philosophique. Cette dernière butte contre cette opacité à partir d'une résistance éprouvée, à moins qu'elle y reconnaisse phénoménologiquement l'oeuvre transparente en soi de la vie auto-affective qu'elle peut alors indiquer par une contre-réduction radicale, sans pouvoir se substituer toutefois à la vie elle-même. En nous souvenant de cette difficulté méthodologique, on peut dire ici que le "discours" philosophique garde également la capacité de "narrer le pathos", et cette narration constitue ainsi la vocation intime de toute génération culturelle qui exprime la Nuit et son Fond par l'évocation d'images jusqu'à la limite du "pouvoir dire", comme en poésie par exemple.

Car même si la philosophie n'est jamais elle-même la vie, son dire ne reste pourtant possible que dans la vie et par celle-ci. C'est en ce sens qu'elle peut "narrer le pathos" en relevant, comme chez Schopenhauer on outre, la phénoménalisation différente entre Représentation et Volonté, sans souscrire pour autant au schopenhauérisme en tant qu'une nouvelle doctrine métaphy- 
sique pessimiste. ${ }^{1}$ Si Schopenhauer montre que la Volonté n'est justement pas réductible à la phénoménalité objective définie par le schématisme kantien, une phénoménologie radicale ne peut pas seulement relever par la suite, avec Husserl, l'élimination impossible de la subjectivité par une épistémologie de type galiléen. Au delà de cette problématique, elle peut encore poursuivre l'oeuvre de la Krisis husserlienne en deça de la subjectivité constituante qui est l'Ego transcendantal, en montrant en effet que le monde-de-la-vie intersubjectif de la perception suppose encore une vie avant tout "monde": à savoir précisément la culturation du besoin en sa naissance passible même. C'est ici que l'on touche à la légitimation ultime d'une narration-discours tant littéraire que philosophique. Car si c'est toujours la vie qui ne parle que d'elle-même, toute parole humaine - et même divine au niveau d'une Révélation religieuse - n'a de sens que par rapport à cette Parole silencieuse de la Vie en son auto-engendrement absolu.

Silence ne veut pas dire autisme, puisque le pathos en son effectuation concrète ou singulière est toujours un "fait". phénoménologique indéniable, justement le besoin de cette douleur, pulsion ou joie. Contrairement à la négation par la conscience, comme chez Sartre par exemple, la tonalité d'un tel affect-besoin du cogito charnel - qui est sans recul possible en son immédiation totale - ne peut jamais être niée. Cette non-liberté originaire détermine chaque modalité de notre vie phénoménologique, ce qui fait que le concept comme l'image s'enfoncent également en cette immédiation pour en "dire" la "parole" secrète qui se tait en tant que logos du monde. Mais la pulsion-besoin est "là" comme donation ainsi que l'angoisse qui se chargent ensemble du souffrir originel pour en faire un effort-éveil à partir d'une résistance: effort de réflexion philosophique comme aussi de création esthétique pour se décharger de ce poids de la vie qui paraît trop lourd par moments, puisque cette vie est rivée à soi sans distance ou écart intermédiaires. Par cette praxis continuelle, toute génération culturelle est à la fois souffrance et joie en nous, avec leur substance commune chaque fois, car depuis tout temps la création est "dépassement" de cet insupportable d'une Nuit sans borne - pour s'engouffrer pourtant en elle en tant que le Fond de toute chose.

Culture et Besoin ne sont donc pas des "choix" discursifs dans la mesure où pulsion, désir, souffrance et plaisir relèvent d'une auto-donation avant toute théorie et ne cessent jamais, tandis qu'une certaine perspective transcendante reste soumise à l'évolution spécifique de la connaissance dans

${ }^{1}$ Cf. M. Henry, Schopenhauer et l'inconscient, in: J.-P. Bertrand (éd.), Schopenhauer et la force du pessimisme, Monaco, 1988, pp. 85-96; La question du refoulement chez Schopenhauer, in: M. Henry, Phénoménologie de la vie, t. II, pp. 131-146.

2 Pour le christianisme, cf. dernièrement A. Vidalin, La Parole de la Vie. La phénoménologie de Michel Henry et l'intelligence des Ecritures, Paris, Parole et Silence 2006, qui insiste beaucoup sur le rapport entre l'épreuve du corps selon Maine de Biran et l'Incarnation. 
l'ordre historique. De la même façon que le motif de la culture est, en dernière analyse, la génération-culturation de la vie elle-même, de même les réalités affectives ou passibles invoquées sont toujours "données" - indépendamment des choix idéologiques du questionnement pour donner, en outre, un "sens" à la vie. C'est cette auto-donation ininterrompue de la vie elle-même qui entre dans l'élucidation thématique de la philosophie en tant que phénoménologie qui voulait - sous sa forme historique chez Husserl et la plupart de ses successeurs - saisir l'eidos de cette vie par la variation d'images, pour se rendre compte que ces données-en-image restent des noèmes irréels, auxquels une phénoménologie radicale oppose la cogitatio réelle en tant qu'effectuation immanente. Le besoin philosophique, ou son désir métaphysique comme disait Emmanuel Levinas, ${ }^{1}$ n'est concevable qu'à partir de ce pathos pratique de la vie elle-même, de sorte que le discours prêté à cette passibilité constitue, en même temps, la Parole secrète de ce pathos lui-même en tant que motivation profonde. L'ouverture au monde résistant, opérée par ce besoin philosophique en son exécution pratique même, comme le montrait Maine de Biran, est la reprise des re-présentations sans nombre pour n'en retenir que les indices de l'auto-présentation vivante qui devient ainsi génération culturelle par le pathos même que la philosophie reconduit à sa Parousie pure, sans cependant pouvoir la "faire voir". Ainsi, tout en poursuivant son travail d'abstraction et d'analyse, une phénoménologie matérielle de la vie n'est jamais coupée de sa source même qui fait réellement vivre le philosophe, en tant qu'individu concret, en un milieu culturel déterminé sans négliger les autres cultures.

En fait, si la phénoménologie de la vie s'attache toujours aussi au vivre le plus simple et le plus strict au sens transcendantal, aucune relation ou situation humaines ne peuvent lui échapper. Le travail solitaire du philosophe rejoint ainsi la culture en son essence même et, par elle également, la vie sociale dans son ensemble qui n'est pas faite d'abstractions économiques et politiques en dernière analyse, puisque la vie sociale représente l'échange vivant même des individus concrets. Le besoin social est la valeur d'échange produite par le travail subjectif et la consommation qui s'insèrent tous les deux dans une manière culturelle particulière de s'habiller, de se nourrir, de se loger, etc. Par là, la réflexion peut s'exercer sur tout objet résistant, sans perdre le contact avec la pathos de la vie, s'il reste clair qu'une telle phénoménologie, appelée pratique, n'est pas obligée de céder à la modernité qui, quant à elle, est justement construite sur l'abandon programmé de la Vie. La perspicacité d'une telle analyse peut alors constituer plus qu'un espoir contre la "crise de la modernité", car elle renoue avec l'absoluïté de la subjectivité, en tant que principe de toute connaissance et action, à partir de la vie qui ne cesse pas de nous étreindre à chaque instant à partir de son auto-mouvement. 
Force est de constater, en plus, que la vie phénoménologique pure est un "objet" dont la philosophie traditionnelle, même dans son courant nommé "Philosophie de la vie", ne parle presque pas, tandis que la littérature, la peinture et les psychologies de la profondeur surtout, l'aborde en vue d'une génération culturelle. Si donc la phénoménologie de la Vie absolue veut "narrer" le pathos de cette Vie, il faut qu'elle travaille, en bonne partie, contre la tradition philosophique en "déconstruisant" ses concepts et ses catégories mondains, afin de pouvoir rejoindre par l'apparaître pur dans l'art (comme nous l'avons vu avec Maine de Biran et Kandinsky), l'échange du co-pathos ainsi que, par sa propre certitude contre-réductive, la manifestation silencieuse, mais toujours vivante, de l'Affectivité originaire. Par ces approches, avec l'épochè du Transcendant comme l'Exteriorité par excellence, et sans nier la résistance originaire, la "narration" de l'Historialité de cette Vie, en tant que Besoin et Culture, s'inscrit naturellement contre toute forme de Barbarie, car celle-ci constitue, pour ainsi dire, l'anti-historialité de la vie, sans s'expliquer pourtant autrement que par cette vie encore. Car si la barbarie est de tout temps et peut se produire à tout moment au niveau du "refus" de la Vie qui s'affecte en nous sans notre consentement préalable, c'est-à-dire lorsque la volonté de nous défaire de notre subjectivité absolue surgit en nous - cette barbarie représente alors une généalogie hors temps qui est le renversement de l'Historialité à l'intérieur du Passage Besoin/Génération ou Affect/Effort. Surmonter l'obstacle pour continuer la vie qui ne peut être que cette continution même par son essence nous semble être, par conséquent, le thème même de toute narration au sein de la Culture, tandis que la Barbarie essaie de prouver le contraire: l'impossibilité de vivre par les seules ressources pratiques de la Vie qui sont en définitive la joie et la souffrance en leur réciprocité commune. Cette Foi de la vie qui constitue la Certitude même de son étreinte passible traverse toute littérature véritable et exerce une fascination qui fait passer notre besoin à l'acte par l'admiration et l'intropathie.

Narrer le pathos en tant que contre-essence (Gegenwesen) de la barbarie correspond, sur le plan philosophique strict, à la détermination phénoménologique de 1" "être" comme Force et Affect qui constituent pour nous la Parousie de cet être dans la Vie et par elle en tant qu'auto-mouvement de naissance, d'accroissement et de dépassement. C'est ici que nous pouvons encore préciser le rôle de l'imaginaire en tant que fiction culturelle et en tant que réalité culturelle. Dans l'art, c'est en général l'ivresse de la vie qui comble en se dressant contre les hallucinations de l'angoisse où le pathos gouverne directement l'image. Car dans l'angoisse poussée à son extrême, toute référence objective se dissout, de sorte que la projection du pathos dans l'image n'est pas tellement une visibilisation de celui-ci, mais plutôt une tentative d'auto-délivrance. Et, dans cette pro-jection imaginaire, il y a comme une illusion de distanciation possible qui ne se produit jamais réel- 
lement dans la vie immanente, comme nous le savons maintenant, parce que celle-ci est cet écrasement de la vie contre elle-même dans l'Abîme au Fond de chaque Soi. Cependant, même s'il y a cette irréalité noématique dans l'image projetée, il s'y opère une modification à l'intérieur de la vie dans la mesure où cette projection imaginaire, en tant que génération culturelle justement, transforme le pathos du désespoir en celui de la félicité qui sont faits de la même chair phénoménologique, à savoir l'Affectivité.

Par conséquent, quand il n'y a eu qu'un seul projet culturel commun, comme par exemple pendant la Renaissance et à d'autres époques, l'imaginaire esthétique, religieux, etc. connaissait moins de ruptures qu'aujourd'hui. Il n'y avait pas seulement l'écriture pour narrer le pathos, mais toute une ville avec ses bâtiments et monuments, et finalement toute une civilisation avec son "art de vivre", de telle manière que le cosmos entier correspondait à la vie individuelle qui pouvait lire sa destinée en cette culture. C'est une telle lecture qui réconcilie la vie avec le monde et permet justement le passage permanent du besoin à la force ainsi que de l'effort à la satisfaction à partir des résistances. Il ne s'agit pas ici d'idéaliser des époques passées, mais de comprendre qu'un pathos secret peut travailler toute une culture pour lui donner une certaine unité de sensibilité, de même qu'un seul pathos caché forge l'intuition d'une oeuvre ou les concepts et métaphores d'une écriture qui concentre les représentations autour d'un même affect-effort. Mais si dans une culture éclatée comme la nôtre, l'affect n'est même plus admis comme un pouvoir de révélation ou de vérité ontologique d'une valeur au moins égale, sinon supérieure à l'objectivité, il est patent que cet affect se disperse en tout sens ou se perd dans la monotonie des stéréotypes. Par conséquent, si aujourd'hui nous cherchons le fondement réel de nos besoins, ce n'est pas l'être distant et anonyme de Heidegger qui réussira à nous le dire, même s'il a essayé, à ses débuts, d'enraciner la facticité du Dasein dans la vie. La raison en est que tout "Dire" reste lié à la vie en tant qu'affection qui fait immanquablement de l'être un "dévoilement" d'une Force-Affect à la mesure de notre angoisse et de son imaginaire. Rien ne paraît donc plus important à l'avenir que de procurer à la vie une sorte de re-présentation véridique d'elle-même. Et c'est ici que réside l'actualité pratique de la phénoménologie radicale de la vie qui, pour l'instant, nous semble être la seule à poser la problématique de la Vie et de la Culture avec autant de clarté phénoménologique.

Pour conclure, il faut donc dire que le Besoin - qui n'est pas le "sujet" ou l" "individualisme" - révèle les structures mêmes de la Vie, c'est-à-dire la passibilité de l'immersion de cette vie en elle-même, sans pouvoir la quitter sinon par la génération-culturation qui contient toujours la narration du "dévoilement" essentiel: le souffrir pathétique promis à sa transformation certaine dans la joie et par elle. Cette modification à l'intérieur d'une expérience phénoménologique pure - et qui est l'essence de toute expérience 
même - peut être nommée métaphysique, car ce "śalut" de transformation ne dépend d'aucune donnée du monde dans la mesure où la vie ne laisse aucun choix en son autoaffection sans distance qui est ma naissance transcendantale. Mais si cette tension, plus forte que la liberté, n'est pas admise, si elle ne devient pas le passage indiqué à la culture, la force une qui traverse la subjectivité entière sur le plan intellectuel, affectif et charnel devient malaise, peur et traumatisme qui sont des réalités dont l'emprise sur les individus semble se répandre autour de nous. Donner le droit à l'imagination, c'est rendre le droit à la vie contre les seules prescriptions positivistes, afin de faire de notre naissance permanente, dans la vie et par elle, un mobile d'action qui reste nourri par le sentiment fondamental du "Je peux" en tant que potentialité de la vie. L'imaginaire est ainsi l'espace pratique du possible: ce lieu où la vie et le monde se rencontrent par l'ouverture que la narration donne à l'affectivité qui s'affecte elle-même dans tout dire pour qu'il puisse être une parole vivante. Pour cela, il faut justement qu'il n'y ait pas seulement le Logos du monde qui fait voir les étants dans leur horizon ekstatique et temporel, mais que le langage s'affecte avant tout par le pathos intérieur. Ainsi le Besoin renvoie à la Culture et celle-ci ne renvoie pas, de son côté, à un redoublement du monde, à une sorte d'arrière-monde, comme disait Nietzsche, mais à ce Fond d'où naît tout besoin - autrement dit, à l'Abîme de la Vie absolue. Inventer des langages conformes à la respiration de la vie et prêter attention à l'imaginaire comme narration du passible, c'est frayer une voie de libération à la vie à partir du premier Résistant immanent qui est, selon Maine de Biran et Michel Henry, notre corps originaire ou affectif en tant que tel.

Adresse de correspondance:

Rolf Kühn

Heuweilerweg 19

D-79 194 Gundelfingen/Frb.

E-mail: rw.kuehn@web.de www.lebensphaenomenologie.at 


\begin{abstract}
By describing body as original power identical to transcendental life of ego, in accordance with radical or material phenomenology, we can establish that the phenomenological truth of a bodily subjectivity is the immanence of pure self-affection.

From the perspective of Michel Henry's view, the Maine Biran and Kandinsky's analyses show clearly that body potentiality unveils pathos linked with the entire universe and turn it into a living cosmos. This cosmic life, visible in art in general and abstract art in particular, makes us aware that this subjective reality is similar to our own inner strength, as we can clearly demonstrate it by the movement of our hand. Thus, the body itself has access to the world without being reduced to a mere instrument or transcendental mean extraneous to our innermost life.
\end{abstract}

